\title{
ASSISTIVE TECHNOLOGIES FOR EFFICIENT MID-RANGE WIRELESS TRANSMISSION OF ELECTRICITY: A REVIEW
}

\author{
Chandrika Bhatnagar ${ }^{1}$, Ved Prakash ${ }^{2}$, Sonal Dahiya ${ }^{3}$ \\ ${ }^{I}$ M.Tech Student, Electronics and Communication Dept, Amity University, Haryana, India \\ ${ }^{2}$ Asst. Prof., Electronics and Communication Dept, Amity University, Haryana, India \\ ${ }^{3}$ Asst. Prof., Electronics and Communication Dept, Amity University, Haryana, India
}

\begin{abstract}
The intention of this paper is to assist the understanding of Wireless Power Transmission in terms of the various techniques used to realize it. Regardless of the hardships in consummating this technology in day to day life, scientists have contemplated Wireless Power Transmission due to its benefits. Mid-range Wireless Power Transmission has been targeted in the paper due to its usefulness. This review paper will address the innovations made in the field recently that aided in achieving higher efficiency of transmission and longer ranges comprising the use of different coil shapes, metamaterials, resonator arrays, relays, multiple transmitter-receiver system and optimal antenna designing.
\end{abstract}

Keywords: WPT-Wireless Power Transmission, SPS-Solar Power Satellite, HR-WPT-Highly-Resonant Wireless

Power Transmission

\section{INTRODUCTION}

Due to heavy dependency of humans on electronic gadgets nowadays it has become indispensable to find ways to power these gadgets wirelessly so as to make the adoption of gadgets unambiguous. Wires are quite cumbersome to handle at times and cause limitations to usage especially while travelling. These challenges have compelled scientists all around the world to look for alternatives to wires. These restraints gave way to a promising technology called Wireless Power Transmission which could possibly eliminate wires and make our lives wireless. This technology has successfully been used in some areas like charging of electric toothbrush, mobile phones and electric vehicles.

Wireless Power Transmission is a process by which electricity can be transmitted from some source or transmitter to one or more receivers [1]. This can be achieved by many ways which depend on the requirements of the system like the transmission distance, power required etc. Three techniques based upon the possible range of power transmission have been practiced namely inductive coupling-based WPT, magnetic resonant coupling-based WPT and electromagnetic radiation-based WPT.

\subsection{Techniques of Achieving Wireless Power}

\section{Transmission}

The design of WPT device is application specific. It depends on the application what technique is to be applied to achieve WPT. For shorter distances (in millimetre range) magnetic induction is used which makes use of Biot-Savart's law and Faraday's law. For longer distances (extending upto a few meters) resonance is incorporated in the system so as to achieve a higher efficiency. Long range power transmission requires the use of microwaves which can carry power to very long distances like a few kilometres. The techniques used are explained below.

Near-field WPT is achieved when the distance between the transmitter and receiver is within one wavelength and is attained by stationary electromagnetic fields [2]. WPT systems based only upon magnetic induction phenomenon can gain high efficiency (approx 95\%) over very short air gaps (approx 70mm) and are basically called 'contactless' instead of wireless [2]. It carries an advantage of simple designing and is safe hence used widely for applications like electric toothbrush, mobile phone and laptop charging and medical implants etc [1]. Resonance can be incorporated into such systems to achieve higher efficiency over longer distances and is achieved by tuning the frequency of the transmitting circuit that maximizes the field distribution between the transmitter and receiver [1]. Two decisive factors viz the Q-factor and the mutual coupling strength determine the power transmission efficiency [1]. This technique is called magnetic coupling and doesn't demand line of sight and is safe for humans but bears two shortcomings viz sensitivity to misalignment and issue of frequency adjustment when charging of multiple devices [1].

Far-field methods are used for achieving WPT for distances longer than one wavelength by the use of propagating electromagnetic fields. Lack of strong coupling leads to much lower efficiencies. Electromagnetic radiation can carry power to longer distances i.e. beyond several meters. But this technique demands commitment of line of sight and beam focussing. Laser beam which are highly focussed with system size being small also serves as a means to carry power to longer distances but has lower generation and 
rectification efficiency [1]. Table 1 summarizes the advantages and disadvantages of the aforementioned basic techniques.

Table 1: (adopted from [1]) Summary of advantages and disadvantages of Wireless Power Transmission techniques

\begin{tabular}{|l|l|l|}
\hline $\begin{array}{l}\text { WPT } \\
\text { Technology } \\
\text { Category }\end{array}$ & Advantages & Disadvantages \\
\hline $\begin{array}{l}\text { Inductive } \\
\text { Coupling }\end{array}$ & $\begin{array}{l}\text { Simple, safe and } \\
\text { higher transfer } \\
\text { efficiency for } \\
\text { short distances }\end{array}$ & $\begin{array}{l}\text { Short } \\
\text { transmission } \\
\text { distance, needs } \\
\text { accurate } \\
\text { alignment }\end{array}$ \\
\hline $\begin{array}{l}\text { Magnetic } \\
\text { Resonant }\end{array}$ & $\begin{array}{l}\text { Long } \\
\text { transmission } \\
\text { distance, no } \\
\text { radiation }\end{array}$ & $\begin{array}{l}\text { Difficulty in } \\
\text { adjusting } \\
\text { resonant } \\
\text { frequency for } \\
\text { multiple devices }\end{array}$ \\
\hline $\begin{array}{l}\text { Electromagnetic } \\
\text { radiation }\end{array}$ & $\begin{array}{l}\text { Very high } \\
\text { transmission } \\
\text { efficiency over } \\
\text { long distances }\end{array}$ & $\begin{array}{l}\text { Harmful } \\
\text { radiation, } \\
\text { demands line of } \\
\text { sight }\end{array}$ \\
\hline
\end{tabular}

\subsection{Brief History of Wireless Power Transmission}

The idea of transmission of electricity without using wires originated almost a century ago, soon after wires were started being used for the transmission of electricity. American scientist Nikola Tesla was the one to perform the first experiments in the field of Wireless Power Transmission which dates back to 1891. His works particularly involved incorporating resonance into his system [2].

During World War II developments were made in the field of microwave which led to the use of microwaves as a means to transmit power. Radiative field or far-field methods were made practical for the first time. In 1960 long-distance power transmission was achieved by Brown [2]. Brown was the first one to coin the term 'rectenna' which could convert microwaves to DC power. Some developments happened to commercialize this technology. However, this was not realistic as microwaves could harm biological organisms. Efforts are being made to commercialize this technology for long distance communication. JAXA (Japanese Space Agency) is developing a full-scale SPS system and it is expected to get completed by 2040[2]. NASA is also reconsidering the technology under its 2001 Space Solar Power Exploratory Research and Technology Program (SERT) [2]. WPT using coupled magnetic resonance regained spotlight when in 2007 Soljacic et al. at MIT performed an experiment based on the concept of magnetic induction to transfer power to a $60 \mathrm{~W}$ bulb at a distance of 2 meters with an efficiency of approximately 40-50\% [3]. Their works had a striking resemblance to the works of Tesla a century ago. This was a key development in the field of mid-range WPT.

\section{HIGHLY RESONANT-WIRELESS POWER TRANSMISSION}

The basic concept of WPT is coupling of electromagnetic fields of the transmitter and receiver antenna. Energy gets transferred from the source to the device when the magnetic fields of the two interact [2]. But with ordinary magnetic induction the range up to which power transfer is possible is low and efficiency is decreased significantly when the two coils are misaligned [1].

Resonance is a property that exists in many physical systems and it can be explained as a natural frequency of the system at which if the system oscillates maximum energy transfer takes place. This property when incorporated in WPT systems increases the transfer efficiency. Without resonance i.e. with ordinary magnetic induction a slight coil misalignment leads to rapid decrease in the transfer efficiency but Resonant Magnetic Coupling gives higher efficiencies over larger distances [3]. Frequency tuning and impedance matching both enhanced the efficiency of power transfer. This made WPT realizable. This technology has been greatly considered by scientists across the world and there's still much room for further advancements. This intention of this paper is to present the developments that took place in this field to further increase the transmission distance and efficiency.

\subsection{System Design}

The basic system design [4] needed for mid-range WPT is shown in figure 1. It shows that the power source is either the AC mains which needs to be converted into DC power or it can be some DC source like a battery. A power factor correction stage might also be needed for high power applications. A high efficiency switching amplifier is employed to convert DC to RF voltage which is needed to drive the resonator. An Impedance Matching Network (IMN) is used to couple the output of the amplifier with the source resonator. It transforms the source resonator impedance loaded by the inductive coupling to the device resonator and the output load. The magnetic field produced by the source resonator excites the device resonator and causes the energy to build in it and this energy is coupled out of the device resonator to be used to power the load directly or charge the battery. For loads requiring DC power a rectifier has been employed to convert AC back into DC. A second IMN may be used here to efficiently couple energy from the resonator to the load.

\section{Source Electronics}

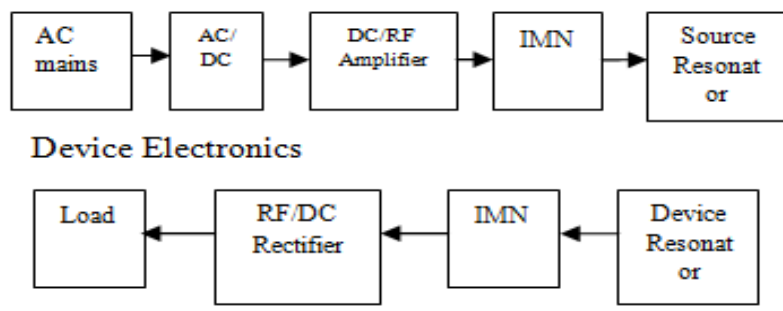

Fig 1: Basic block diagram of HR-WPT (adopted from [4]) 


\subsection{Recent Developments in HR-WPT}

Moorey et al. have analysed of the basic techniques used for WPT [2]. He has presented a detailed description of the applications and the type of technology used in that application. He has also presented the modelling techniques of WPT and the general model for WPT with a conclusion that geometry is the important thing to consider for efficient WPT system.

\subsubsection{Metamaterials and Resonator Array for HR-}

\section{WPT}

Karthick et al. have studied two major techniques to enhance the efficiency of power transmission and the range of WPT viz 'metamaterials' and 'array of resonators' [5]. Metamaterials are artificial materials having negative refractive index. They act as a super lens when placed between two resonators and are able to enhance the coupling efficiency and thus the overall power transmission efficiency. A slab of material with negative permittivity and permeability can enhance both near- and far-fields. It was shown by numerical simulations that a slab of metamaterial with permeability as $-1+0.05 \mathrm{i}$ and permeability as 1 enhances the field intensity and hence acts as a super lens. In WPT the ratio of wavelength to size of unit artificial structure should at least be greater than 100 .

In an experiment metamaterial was formed by two-sided square spirals by printing 3-turn spirals on Rogers RO4003C circuit board, with two sides connected by wires. With a size of $6.5 \times 6.5 \mathrm{~mm}$ the structure gave strong response to external magnetic field around $24 \mathrm{MHz}$ resonant frequency. It was found that at above resonant frequency metamaterials give less power transmission efficiency improvement. It was found that at a distance of $50 \mathrm{~cm}$ between the resonating coils the efficiency without metamaterial slab was only $17 \%$ whereas it enhanced to $35 \%$ by using the slab.

The design of metamaterial slab was simplified and an anisotropic slab was constructed using only two flat panel spirals and by experiments it was found that efficiency increased from $17 \%$ to $47 \%$ by using the slab. By experiments it was found that metamaterials are also capable of lighting a $40 \mathrm{~W}$ bulb i.e. they are capable of handling high power.

An array or resonators was used by Karthick et al. [5] to increase the range of power transmission and also the increase of efficiency for moving vehicles. A system of 10 resonators which were square spirals of width $20 \mathrm{~cm}$ was modelled and simulated using COMSOL with resonant frequency being $25 \mathrm{MHz}$. The field distributions were calculated and plotted and were found out to be nonuniform. It was also found that more number of receivers for a single transmitter resonator gives higher overall efficiency where overall efficiency is the sum of the individual efficiencies. Different efficiencies could be achieved at same position for different frequencies and to optimize the power transfer in real system a feedback system could be employed to adjust the transmitting frequency depending upon the feedback provided by the receiver to the transmitter.
An experiment was demonstrated by modifying a toy train set and running it on an oval track. A total of 6 square and 24 trapezoidal shape resonators were used to cover the track both having a resonating frequency of $25 \mathrm{MHz}$ A square antenna was used at transmitting end and a square resonator was connected to a $40 \mathrm{~W}$ bulb as a receiver. Methods to improve the performance of array system had also been discussed.

\subsubsection{Powering Moving Vehicles using Array of}

\section{Resonators}

Yerazunis et al. demonstrated by an experiment that power can be transferred to moving vehicles by the use of arrays [6]. They used a toy train and two different tracks one of which was oval and the other one straight with multiple square and trapezoidal resonators for the experiment. He found that power can be transferred to the train with minimal local energy storage needed but due to resonant nulls in the frequency he used i.e. $23-25 \mathrm{MHz}$ power fluctuations were observed. These fluctuations could be handled by frequency control techniques.

Srinivas et al. have investigated various methods of charging electric vehicles namely Inductive Power Transfer and Magnetic Coupled Resonance [7]. He has focussed on safety considerations in Witricity charging highlighting the issues of overcharging of batteries, misalignment of coils and field exposure. He has designed control circuitry to address these issues

\subsubsection{Various Coil Shapes to Enhance Efficiency}

Karthikeyan et al. tested various coil shapes for their efficiencies and transmitted output power [8]. He tested by experimenting with circular, rectangular and multicore circular coils. The distance between the transmitter and receiver coils is changed for all three coils and the graph showing their efficiencies and output power as a function of distance is plotted using MATLAB. He found out that circular twisted pair of coils gives better performance as compared to other coils.

McDonough and Fahimi studied through simulation about how the geometry of coils can affect the maximum power transferred and cost of the system [9]. They studied the square and circular, air core, 'pad-type', coupled inductors with circular pad being $1 \mathrm{~m}$ in diameter and square coil being $1 \mathrm{~m}$ in width having 1 turn each. Tolerance to misalignment was studied by offsetting the coils between 0 $0.5 \mathrm{~m}$. They found that the circular coil operates better when perfectly aligned but with slight misalignment the mutual inductance droops off faster than the square coil. And that energy storage requirement for both the coils depends heavily on number of turns of the coil.

\subsubsection{Use of Relay to Increase Efficiency}

Wang et al. investigated the optimal position of a relay in a transmitter and receiver coil system using mathematical analysis and performing experiment and found that the 
optimal position of relay depends on the system load [10]. It is not supposed to be in the middle of the transmitter and receiver. The team used a three resonator system containing spiral resonator having a diameter of $2.6 \mathrm{inch}, 0.11$ inch trace width and 0.06 inch trace spacing. The resonant frequency measured was $29.5 \mathrm{MHz}$ with Q-factor of 110 and intrinsic loss of $0.2 \mathrm{Mrad} / \mathrm{s}$. The efficiency was measured for different relay positions and load values. It was found that with the increase of load value efficiency also increased and the relay resonator was required to move towards the device resonator to achieve maximum efficiency.

\subsubsection{Multiple Transmitters and Receivers System}

Nguyen et al. studied a Multi-Input Multi-Output WPT system was presented in which they found out the efficiency of power transfer both theoretically and experimentally [11]. An intermediate repeater antenna was included in a MIMO system comprising two transmitters and two receivers and it was found that the repeater enhances the overall system efficiency. Transistor IRF510 (Fairchild) was used in class$\mathrm{E}$ amplifier to generate a working frequency of $2 \mathrm{MHz}$. Some setups were made and after all experiments system efficiency was found to be $90 \%$ with impedance matching. With different setups it was also found that optimum number of transmitters is a trade-off result for maximum system efficiency in a MIMO system.

\subsubsection{Optimal Antenna Designing}

Rajaraman et al. proposed a miniaturized dual-band miniaturized patch antenna with improved gain and a compact structure as compared to standard microstrip patch antenna [12]. The compact size has an obvious advantage over larger sized antennas when talking of portable electronic devices. A Rectangular Inset fed microstrip patch antenna is designed to resonate at $2.86 \mathrm{GHz}$. This is then loaded with two pairs of CSRRs(Complementary Split Ring Resonators) designed to have very different resonant frequencies towards the right and left sides with size of $7.1 \mathrm{~mm}$ and $9.3 \mathrm{~mm}$ respectively. The reactive impedance surface with dimensions and spacings well adjusted to increase the gain of the antenna is used. The designing and simulation is done using HFSS. Both the variants designed show good efficiencies with acceptable return loss performance with the second variant giving the highest efficiency of $81.8 \%$ at $2.39 \mathrm{GHz}$. This work was targeted to increase gain values for linear polarization and in future similar efforts could be made for circular polarization.

\section{CONCLUSION}

Wireless Power Transmission technology is indeed a promising solution to the problems aroused by the existing technology. Out of the many ways of achieving WPT 'magnetic resonant coupling' is the most examined method for mid-range power transfer. Despite this the technology has not been publicly accepted due to limitation of range and safety considerations but attracts the diligence of researches due to its advantages. Efficiency and range of transmission enhancement are the areas in spotlight for making improvements and are contemplated by scientists. Metamaterials have improved the efficiency to three times of that achieved without using them. The use of resonator arrays has increased the range of power transmission. Use of relays has also contributed to efficiency enhancement. The miniaturized dual-band patch antenna also gave higher efficiency. The geometry of coils can be modified to achieve higher efficiencies. There's still much room for improvements in this field.

\section{ACKNOWLEDGEMENTS}

I am very thankful to my faculty members Mr. Ved Prakash and Ms. Sonal Dahiya who contributed towards my work.

\section{REFERENCES}

[1]. Xiaolin Mou and Hongjian Sun, "Wireless Power Transfer: Survey and Roadmap", Proceedings of IEEE VTC 2015 Spring Workshop on ICT4SG, 18 Feburary 2015

[2]. Charles L. Moorey, Willliam Holderbaum and Ben Potter 2014. "A Review of Modelling Techniques Used In The Analysis of Wireless Power Transfer systems," ELECTRONICS, VOL. 18, NO. 2, DECEMBER 2014

[3]. Witricity-The Basics, available at http://witricity.com/technology/witricity-the-basics/ accessed on $4^{\text {th }}$ Jan 2015

[4]. Dr. Morris Kesler 2013. "Highly Efficient Wireless power Transfer: Safe, Efficient, and over Distance," Witricity Corporation, 2013 available at http://www.witricity.com/assets/highly-resonant-powertransfer-kesler-witricity-2013.pdf/ accessed on $4^{\text {th }}$ Jan 2015 [5]. K.karthick, A Anandhkumar, R Sonal, "Wireless Power Transfer: Metamaterials and Array of Coupled Resonators" [6]. Yerazunis, W., Wang, B. and Teo, K.H. 2012. "Power Delivery Optimization for a Mobile Power Transfer System based on Resonator Arrays," IEEE International Symposium on Antennas and Propagation (ISAP), October 2012

[7]. Adarsh Srinivas, Deepak Gadodia, Sahil Kadam and Vaibhav Chauhan 2014. "Car Park Charger Using Witricity," International Journal of Engineering Trends and Technology (IJETT) - Volume 16 Number 5 - Oct 2014

[8]. R.Karthikeyan, P.Mahalakshmi, N.GowriShankar and S.Elangovan 2014. "Performance Evaluation of Wireless Power Transfer through Various Coil Shapes," International Journal of Advanced Research in Electrical, Electronics and Instrumentation Engineering, Vol. 3, Issue 10, October 2014 [9]. Matthew McDonough and Babak Fahimi, "Comparison between circular and square coils for use in Wireless Power Transmission," University of Texas, Texas, USA

[10]. Hao Wang, Zhi-Hong Mao,Qi Xu, and Mingui Sun, "Relay Effect on Wireless Power Transfer Using Resonant Coupling Hao"

[11]. Minh Quoc Nguyen, Dakota Plesa, Smitha Rao, and J.c. Chiao 2014. "A Multi-Input and Multi-Output Wireless Energy Transfer System,” 978·1-4799-3869-8/14®2014 IEEE

[12]. Gayathri Rajaraman, M. Anitha, Athrish Mukerjee, Khagindra Sood and Rajeev Jyoti 2015. "Dual-Band, Miniaturized, Enhanced-Gain Patch Antennas using Differentially-Loaded Metastructures," Indian Journal of Science and Technology, Vol 8(1), 11-16, January 2015 